\title{
SEIDE IST WIE NICHTS UND NICHTS IST WIE SEIDE
}

\section{Liebe Kolleginnen und Kollegen!}

Seide steht - wie kein anderer Begriff - seit dem Altertum als Synonym für China. Seide heißt in der chinesischen Sprache ,si“", davon abgeleitet war in der Antike die Bezeichnung „Seres“ für China geläufig, die Bewohner des Seidenlandes wurden „Serae“, also Seidenleute genannt. Das mittellateinische Wort für Seide lautet „Seta“. Die heutige Seide stammt ausschließlich von Bombyx mori, dem domestizierten weißen Maulbeerspinner, der sich wie der Name sagt - ausschließlich von Maulbeerblättern (Morus) ernährt. Der Ursprung der Seidenraupenzucht in China geht der Legende nach auf die 3 legendären Urkaiser Fu Xi, Shen Nong und Huang Di zurück, welche um das 3. Jahrtausend v.Chr. gelebt haben sollen. Die Gattin des Gelben Kaisers Huang Di namens Xi Ling, auch Leizu genannt, soll dem Volk die Zucht von Seidenraupen, die Gewinnung von Seidenfäden durch Abhaspeln von den Kokons und das Weben von Seidenstoffen beigebracht haben. Sie wird heute noch als "Seidenraupengöttin“ (Cangu shengmu) verehrt. Anhand dieser Chronologie der Urkaiser errechnete man für die Entstehung der Seide eine Zeit zwischen 2700 und 2600 v.Chr. Bei Ausgrabungen in Qianshanyang in der Provinz Zhejiang wurde Fragmente von Seidenstoffen gefunden - und siehe da! Diese konnten mittels Radiocarbondatierung tatsächlich auf die Zeit um 2650 v.Chr. datiert werden.

Seidenfasern sind extrem reißfest, angeblich 5-mal so fest wie die Kunstfaser Kevlar, aus welcher kugelsichere

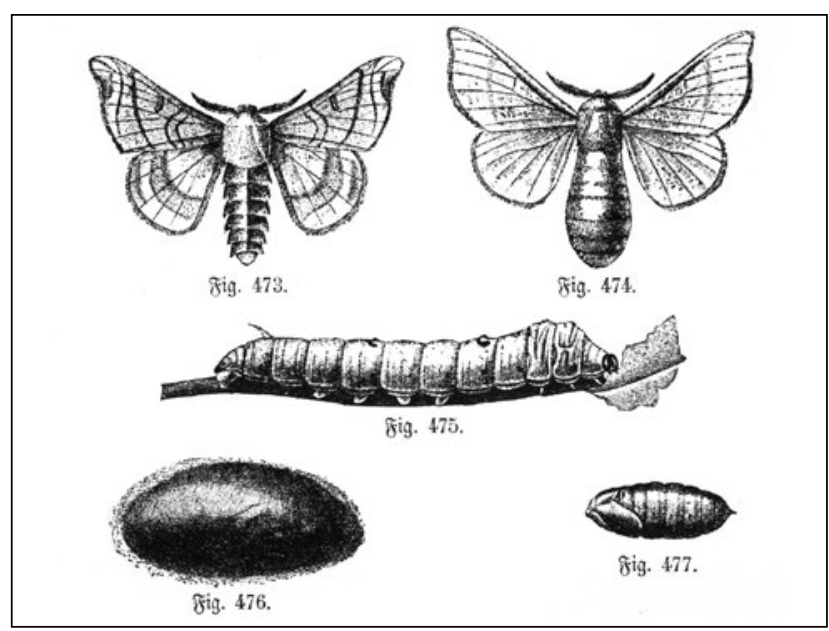

Abb. 1: Der Seidenspinner Bombyx mori in seinen Entwicklungsstufen: adulte Form (links männlich rechts, weiblich), Seidenraupe, Kokon, Puppe (aus: Schulnaturgeschichte 1891, Hubert Ludwig (C) Gemeinfrei)
Westen gefertigt werden. Der Seidenspinner ist ein ca. 38 $\mathrm{mm}$ großer, weißer oder perlgrauer Nachtfalter aus China. Nach der Paarung, die bis 12 Stunden dauern kann, stirbt das Männchen (Kunststück!) und die Weibchen legen 400 Eier. Nach dem Überwintern schlüpfen die Seidenraupen, englisch silkworm genannt, welche sich insgesamt 4-mal häuten und dieser Zeit das 40.000-Fache ihres Körpergewichts an Maulbeerblättern fressen. Von $2 \mathrm{~mm}$ wachsen die Räupchen dank ihrer Fresslust innerhalb eines Monats auf eine Länge von 9-10 cm. Die Schmatzgeräusche in einer Zuchtanlage tönen wie ständiger Regen. 30-35 Tage nach dem Ausschlüpfen aus dem Ei beginnt die Raupe mit der Produktion der aus Proteinen bestehenden Seidensubstanz. Aus der an der Unterlippe gelegenen Spinnwarze tritt der Seidenfaden aus, der sich sofort an der Luft erhärtet. Mit gezielten Kopfbewegungen wickelt die Raupe Windung um Windung in 8er-Touren von außen nach innen um sich herum, bis sie vollständig von einem dichten Seidengespinst, dem Kokon eingehüllt ist (Abb. 1).

Nach 8 Tagen schlüpft der fertige Schmetterling, indem er den Kokon durchbeißt, was natürlich die Qualität der Seide beeinträchtigen würde. Um das zu verhindern, werden die Kokons in kochendes Wasser geworfen, was gleichzeitig die Puppe abtötet und den Klebestoff auflöst, der die Fäden zusammenhält. In der modernen Alternative stirbt die Raupe in $30 \mathrm{sec}$ bei $120^{\circ} \mathrm{C}$ in der Mikrowelle. Dies ruft Tierschutz-Organisationen wie PETA (People for Ethical Treatment of Animals) auf den Plan, welche gegen den modebedingten „Massenmord“ von Lebewesen protestieren. Vom Kokon der Seidenraupe kann ein endloser (bis $4000 \mathrm{~m}$ langer) Seidenfaden abgewickelt werden, für ein Seidenkleid braucht es 1700 Kokons. Die Aufzucht der Seidenraupen ist eine heikle Angelegenheit: Im alten China wurden sie in peinlich sauberen, wohl temperierten Räumen gehalten, sie erhielten 48 Mahlzeiten täglich aus kleingeschnittenen Maulbeerblättern von Pflegerinnen, die auf Zehenspitzen gingen und flüsterten, wenn sie Fliegen vertrieben und kranke Exemplare, verdorbene Blätter oder Kot aussortierten. Menschen, die mit den Raupen zu tun hatten, aßen nichts, was Blähungen verursachte. Weder Menstruierende noch Schwangere noch parfümierte Damen durften zu den Tieren, auch Leute in tiefer Trauer waren wegen ihrer bedrückenden Ausstrahlung nicht willkommen. Ein völlig anderes Szenario spielte sich in der Mitte des 18. Jahrhunderts im Hause Goethe in Frankfurt 
ab: In seinen Jugenderinnerungen „Dichtung und Wahrheit" beschreibt Johann Wolfgang von Goethe ein Kindheitstrauma: Sein Vater Johann Caspar Goethe versuchte sich in der Zucht von Seidenraupen. In einem Mansardenzimmer waren Tische und Gestelle aufgestellt für die schnellwachsenden heißhungrigen „Seidenwürmer“, für welche man kaum genügend Blätter herbeischaffen konnte. Goethe schrieb, dass die Kinder (seine Schwester Cornelia und er) diese Arbeit bei günstiger Witterung als lustige Unterhaltung ansahen, bei Kälte und Nässe jedoch „machte es große Not, denn diese Geschöpfe können die Feuchtigkeit gar nicht vertragen und so mussten die benetzten Blätter sorgfältig getrocknet werden. Mancherlei Krankheiten haben die armen Kreaturen zu Tausenden dahingerafft." Die entstehende Fäulnis habe pestartig gestunken und weil man die Toten und Kranken von den Gesunden absondern und wegschaffen musste, war dies „in der Tat ein äußerst beschwerliches und widerliches Geschäft" gewesen, „das uns Kindern manche böse Stunde verursachte.“

Mehr als 3000 Jahre lang besaß China eine absolute Monopolstellung in der Seidenproduktion. Seit dem 1.Jahrhundert v.Chr. erfolgte aufgrund der hohen Nachfrage nach chinesischer Seide ein reger Handel mit dem Römischen Reich zu Wasser und zu Lande. Da ein Kilogramm Seide mit einem Kilogramm Gold aufgewogen wurde, verursachte der Import chinesischer Seide einen erheblichen Abfluss an Goldreserven des römischen Imperiums. Abgesehen davon wurde das Tragen von Seidenkleidern als dekadent und unmoralisch angesehen. Der Philosoph Seneca sagte in den Declamationes: ,Ich kann Seidenkleider sehen, sofern Stoffe, die weder Körper noch Anstand verbergen, überhaupt Kleider genannt werden können. [...]

Erst gegen Ende der Spätantike gelangte das Geheimnis der Seide zum oströmischen Kaiser Justinian I. (482-527 n.Chr.): 2 Wandermönche schmuggelten im Jahr 522 n.Chr. Eier des Seidenspinners und Maulbeersamen in ihren Bambusstöcken nach Byzanz. Araber gründeten im 8. Jahrhundert Seidenwerkstätten in Spanien, von dort gelangte die Kenntnis der Seidenproduktion nach Sizilien, wo ab dem 12. Jahrhundert Palermo und Messina wichtige Zentren der Seidenindustrie waren. Im 13. Jahrhundert verdankte die Stadt Lucca in der Toskana ihren Einfluss den Wasser- kraft-getriebenen Seidenzwirnmühlen und der Farbenpracht der Luccaner Seide. In Frankreich war die Nachfrage nach Seide so groß, dass im Jahre 1470 König Louis XI. eine eigene Seidenproduktion aufbauen ließ und der Stadt Lyon dazu das französische Monopol verlieh. Er machte sie damit zum Zentrum der Seide für ganz Europa. Neben Lyon und Zürich etablierte sich auch eine bedeutende Seidenindustrie in der Stadt Krefeld in Westfalen, welche durch die Familie von der Leyen dominiert wurde.

Im Jahr 1854 wurde praktisch der ganze Seidenraupenbestand Europas von der Fleckenkrankheit befallen. Das war das Ende der Seidenproduktion nördlich der Alpen. Ein neuer Anstoß erfolgte um 1856, als der Engländer William Henry Perkins den ersten synthetischen AnilinFarbstoff erfand, der in seiner Heimat gar nicht, aber in Lyon durchaus auf großes Interesse bei den Seidenfärbern stieß. Bald wurden überall Anilin-Farbenfabriken gegründet: die Farbwerke Hoechst, die Badische Anilin- und Sodafabrik BASF oder die Aktiengesellschaft für AnilinfarbenFabrikation AGFA in Deutschland. In Basel gründete der Seidenweber Alexander Clavel aus Lyon die Chemische Industrie Basel CIBA. So ist praktisch die ganze Pharmaindustrie in Europa aus der Seidenfärberei entstanden.

Der Begriff „Seidenstraße“ wurde vom deutschen Grafen und Geografen Ferdinand von Richthofen geprägt, der ihn 1877 erstmals verwendet hat.

Ein Netz von Karawanenwegen führte über $6400 \mathrm{~km}$ von der alten Hauptstadt der Qin-Dynastie Xi'an entlang der Chinesischen Mauer nach Nordwesten, durchquerte die Wüste von Taklamakan, überquerte das Pamirgebirge und führte über Afghanistan und Persien bis ans Ostufer des Mittelmeers. Zwischen 100 v.Chr. und dem 13. Jahrhundert erlangte die Seidenstraße ihre größte Bedeutung. Unter dem jetzigen Staatspräsidenten Xi Jinping wird die „Neue Seidenstraße“ unter der Bezeichnung „One Belt, One Road" als Handelsoffensive in Form eines interkontinentalen Handelsnetzes zwischen derVolksrepublik China und 60 weiteren Ländern in Afrika, Asien und Europa propagiert.

Ihr

A.Wirz-Ridolfi

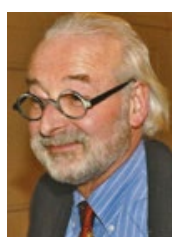

Dr. med. Andreas Wirz-Ridolfi

Chirurgie FMH, Akupunktur/TCM

Medi-China $A G$,

Im langen Loh 11, 4054 Basel/Schweiz

Tel.: +41613016585 